\title{
The Multiple-Camera 3D Production Studio
}

\author{
Jonathan Starck*, Atsuto Maki, Shohei Nobuhara, Adrian Hilton, Takashi Matsuyama
}

\begin{abstract}
Multiple-camera systems are currently in development as a means to capture and synthesise highly realistic three-dimensional (3D) video content. Studio systems for 3D production of human performance are reviewed from the literature and the practical experience gained in developing prototype studios is reported across two research laboratories. System design should consider the studio backdrop for foreground matting, lighting for ambient illumination, the camera configuration for scene capture as well as accurate geometric and photometric camera calibration. A ground truth evaluation is performed to quantify the effect of different constraints on the multiple camera system in terms of geometric accuracy and the requirement for high quality view synthesis. Camera height has only a limited influence on surface visibility, multiple camera sets or an active vision system can be required for wide area capture, accurate reconstruction requires a camera baseline of 25 degrees and the achievable accuracy is $5-10 \mathrm{~mm}$ at current camera resolutions. Accuracy is inherently limited and view dependent rendering is required for view synthesis with sub-pixel accuracy where display resolutions match camera resolutions. The two prototype studios are contrasted and state-of-the-art techniques for 3D content production are demonstrated.
\end{abstract}

\section{INTRODUCTION}

Since the pioneering work of Kanade et al. [1] introduced the capture of a 3D Virtualized Reality from multiple view video, there has been extensive research on the 3D reconstruction and rendering of people from video images. People form a central element of media content and multiple camera acquisition of a performance provides interactive control of a virtual viewpoint to create $3 \mathrm{D}$ video, termed free-viewpoint video. Marker-based camera capture is already widely used in production for feature films and computer games through either optical or electromagnetic marker systems. This technology allows the motion of an actor to be replayed on a computer generated character but is inherently limited to the acquisition of

J. Starck and A. Hilton \{j.starck, a.hilton@surrey.ac.uk\} are with the Centre for Vision, Speech and Signal Processing, University of Surrey, UK. Tel: +44-1483-686030 Fax: +44-1483-686031

A. Maki, S. Nobuhara and T. Matsuyama \{maki, nob, tm@i.kyoto-u.ac.jp\} are with the Graduate School of Informatics, Kyoto University, Japan. Tel: +81-75-753-4891 Fax: +81-75-753-4769 
a sparse set of 3D marker points and does not provide detailed information on body or cloth movement or appearance. Markerless performance capture, a virtualisation of reality, provides the detail of a live performance through 3D video production.

Research to-date has focused on studio-based multiple camera acquisition systems and the computer vision algorithms required to achieve robust reconstruction and high-quality view synthesis either as a real-time or off-line post-process. Studios have been developed using different designs in terms of physical layout, backdrop, lighting, camera system and the choice of algorithms used for 3D reconstruction and view synthesis. This paper brings together the experience of two research laboratories developing prototype multiple-camera systems for 3D production. At the University of Surrey in the UK a freeviewpoint video system [2] has been developed to create highly realistic animated 3D digital characters. At Kyoto University in Japan, a free-viewpoint video system [3] has been developed to capture the shape, appearance and motion of intangible cultural assets for storage, transmission and 3D display.

Three contributions are made in this paper.

- Practical design considerations are identified for a multiple camera capture system.

- A ground-truth evaluation is presented to provide an insight into different design decisions.

- A comparison is provided for 3D production of human performances in two real studios.

Section II describes related work and provides an overview of systems designed to capture the wholebody shape and appearance of people. Practical issues in designing a multiple camera capture studio are then presented in Section III. A ground-truth evaluation is presented in Section IV using a synthetic data-set to assess the effect of geometric accuracy on the camera system design. A practical comparison is then presented in Section V for two different studios currently in use for human performance capture and 3D video production. Finally, Section VI draws conclusions on the design and quality of media production in the multiple camera 3D Production Studio.

\section{RELATED WORK}

Studio-based multiple-view capture of 3D events has been researched in both computer vision and computer graphics. In computer vision, markerless human motion tracking and image-based shape reconstruction have been investigated. In computer graphics, model building is performed for both static and dynamic shapes, and image-based rendering has been investigated for view synthesis. Recent advances have seen the convergence of computer vision and computer graphics techniques, which has achieved offline production of free-viewpoint video with a visual quality comparable to the captured video. In 
this section we summarise the related work in which multiple camera studio technology is used for 3D capture and rendering.

\section{A. Model-based shape capture}

Markerless human motion capture from single and multiple view video has been a central topic in computer vision research over the past decade with over 500 papers published in this field in the past five years alone [4]. Markerless tracking has been combined with shape reconstruction to capture the changing shape, appearance and motion of a parameterised model from multiple view video. Starck and Hilton [5] describe a shape optimisation process to match an articulated surface mesh to multiple shape cues across camera images. Carranza et al. [6] track a generic humanoid model and adapt the shape to match multiple silhouette images. Theobalt et al. [7] use the model geometry as an efficient means to encode multiple view images for transmission and 3D display. Sand et al. [8] capture the shape of the human body under articulation using multiple silhouette images. Balan et al. [9] derive a parametric model of shape and pose-dependent deformations from a database of range scans of human bodies to match human shape and pose in multiple views. Model-based techniques introduce prior knowledge to constrain 3D geometric reconstruction in the presence of ambiguities and also provide a single consistent model structure that would allow the analysis of shape deformations for example in coding and transmission or re-use in animation. However, model-based techniques are inherently limited to a transformation of the predefined model and cannot be applied to complex scenes with large changes in structure such as loose clothing or hair on the human body.

\section{B. Image-based scene Reconstruction}

Image-based 3D scene reconstruction without a prior model is a key problem in computer vision. Seitz et al. [10] provide a quantitative evaluation of state-of-the-art techniques using multiple camera views. Conventional stereo-based techniques reconstruct a $2.5 \mathrm{D}$ depth image representation from two or more cameras through a regularized search for image correspondence. Stereo reconstruction suffers from ambiguities in 2D image matching with uniform surface appearance, depth discontinuities and unknown surface visibility. Volumetric reconstruction techniques instead derive the $3 \mathrm{D}$ volume that is consistent with multiple images. A volume representation allows inference of visibility and integration of appearance across multiple widely spaced camera views. Shape-from-silhouette (SFS) techniques derive the visualhull, the maximal volume that is consistent with a set of foreground images [11]. However, the visual-hull only provides an upper bound on the volume of the scene, concavities that are occluded in silhouettes 
are not reconstructed, appearance is not matched across images and phantom volumes can occur that are consistent with the image silhouettes. Space-carving techniques [12] provide the photo-hull, the maximal volume that is photo-consistent across all visible camera images. Independent voxel carving however suffers either from under or over carving according to the photo-consistency criteria. Regularisation has been introduced using a level-set approach to multiple view stereo [13], [14]. Multiple shape cues have been combined for robust reconstruction using iterative local-surface optimization techniques. The visualhull [3], [15], [16], [17] is often used to provide an initial surface, however optimization is subject to local minima and the surface is constrained to represent only those structures that are initially defined and can retain incorrect structures.

\section{Video-based Rendering}

Image-based rendering is the process of synthesising novel views from camera images. With no geometric scene information, synthesis is performed directly by resampling multiple view images as a set of samples from the light-field in a scene [18]. Highly realistic view synthesis can be achieved at the cost of requiring dense image sampling to avoid artefacts in interpolating images without exact image registration. Image-based modelling and image-based rendering have been combined [19], [20] to synthesize novel views from a sparse set of cameras by using scene geometry to provide the correspondence between images. The advantage of view-dependent rendering is that it can overcome inaccuracies in geometric scene reconstruction by reproducing the change in surface appearance that is sampled in the original camera images. However, with inexact geometry and errors in camera calibration the correspondence between camera images can be inexact leading to blurring and double exposure effects [16]. Zitnick et al. [21] demonstrate view synthesis approaching the quality of the original camera images using stereo reconstruction to provide the underlying scene geometry with a narrow camera baseline.

\section{Studio Capture Systems}

Camera systems developed for human shape reconstruction and video-based rendering can be characterised by the nature of the reconstruction and rendering process together with the target application area. Online systems are based on fast and robust shape reconstruction for real-time shape and appearance in mixed reality environments. Video-rate reconstruction from silhouettes has been achieved using volumetric discretization [22], [24], [25] and polyhedral intersection [23], [26]. Waschbusch et al. [33] present a video-rate structured-light system to actively acquire geometry in cluttered scenes. Offline systems have the potential for more accurate geometric scene reconstruction. Carranza et al. [6], [27] and Sand et al. 


\begin{tabular}{|c|c|c|c|c|c|c|}
\hline Technique & Ref & Cameras & Coverage & Background & Reconstruction & Applications \\
\hline \multirow{5}{*}{ Real-time } & {$[22]$} & 5, QVGA, 24bpp, 30fps & $360^{\circ}$ & Semi-controlled & Silhouettes, $15 \mathrm{~Hz}$ & Visualisation \\
\hline & {$[23]$} & 4, QVGA, 15fps & $180^{\circ}$ & Semi-controlled & Silhouette, $15 \mathrm{~Hz}$ & Visualisation \\
\hline & [24] & 6, PAL, 24bpp, 25fps & $180^{\circ}$ & Retro-reflective & Silhouette, $15 \mathrm{~Hz}$ & On-set Preview \\
\hline & {$[25]$} & 4, VGA, 16bpp, 30fps & $180^{\circ}$ & Semi-controlled & Silhouette, $30 \mathrm{~Hz}$ & Virtual Worlds \\
\hline & {$[26]$} & 6, SVGA, 16bpp, 30fps & $360^{\circ}$ & Semi-controlled & Silhouette, $30 \mathrm{~Hz}$ & Mixed reality \\
\hline \multirow{3}{*}{ Model-Based } & [27] & 8, VGA, 16bpp, 30fps & $360^{\circ}$ & Semi-controlled & Silhouette & 3D Video \\
\hline & {$[8]$} & 3, NTSC, 30fps, 10 mocap & $180^{\circ}$ & White & Silhouette & Modelling \\
\hline & {$[5]$} & 9, PAL, 25fps & $360^{\circ}$ & Blue & Silhouette + Stereo & Modelling \\
\hline \multirow{4}{*}{ Model-Free } & {$[28]$} & 51, NTSC, 16bpp, 30fps & $360^{\circ}$ & Semi-controlled & Stereo & Virtualized Reality \\
\hline & {$[3]$} & 25, VGA, 16bpp, 30fps & $360^{\circ}$ & Grey & Silhouette + Stereo & 3D Video \\
\hline & [29] & 8, VGA, 24bpp, 30fps & $360^{\circ}$ & Beige & Silhouette + Stereo & Modelling \\
\hline & {$[2]$} & 8, HD(1080p), 16bpp, 25fps & $360^{\circ}$ & Blue & Silhouette + Stereo & Animation \\
\hline \multirow{4}{*}{ Short-baseline } & {$[30]$} & 48, QVGA, 15-20fps & $30^{\circ}$ & Natural & Colour & Mobile cameras \\
\hline & {$[21]$} & 8, XVGA, 8bpp, 15fps & $35^{\circ}$ & Natural & Stereo & View-Interpolation \\
\hline & [31] & $16,1300 x 1030,12 \mathrm{fps}, 8 \mathrm{bpp}$ & $30^{\circ}$ & Natural & none & 3D TV \\
\hline & {$[32]$} & $100,1392 \times 1040,30 \mathrm{fps}, 8 \mathrm{bpp}$ & $30^{\circ}+$ & Natural & none & $3 \mathrm{D} \mathrm{TV}$ \\
\hline
\end{tabular}

TABLE I

Multiple CAMERA CAPTURE SySTEMS

[8] adapt a generic model to silhouettes and Starck and Hilton [5] match silhouette and stereo cues. The 'Virtualized Reality' project [1] first reconstructed dynamic scenes of people using a 51 camera dome by fusing multiple $2.5 \mathrm{D}$ stereo depth images into a single 3D surface. Franco et al. [34] derive shape from silhouette, Tomiyama et al. [35], Matsuyama et al. [3] and Starck and Hilton [16] refine shape from silhouette to match stereo cues. Starck and Hilton [2] combine multiple shape cues for robust widebaseline volumetric reconstruction using graph-cuts [36]. Goldluecke and Magnor [14] introduce a spatiotemporal approach to volumetric reconstruction using level-sets for temporally consistent reconstruction. Vedula et al. [37] introduce 3D scene-flow for spatio-temporal interpolation in view synthesis. Finally short-baseline techniques make use of dense camera configurations with a restricted viewing range to achieve high-quality video interpolation for view synthesis [21], [31], [30].

Table I gives a summary of systems and techniques that have been applied to whole-body human shape and appearance capture. While the list is not exhaustive it is provided to cover the breadth of research presented in the area and a comparison of the camera systems. 


\section{Studio System Design}

Designing a multiple camera studio requires a number of decisions to be made in terms of the physical layout of the studio, the backdrop, illumination, and the camera system for 3D capture. In this section the factors affecting different design decisions are summarised.

\section{A. Backdrop}

Foreground matting [38], the process of extracting the foreground objects from an image, is often the first step in reconstructing a 3D dynamic scene from multiple view video. Coloured screens allow relatively simple background removal through a process termed chroma-keying where a known colour or a narrow range of colours is automatically removed. Blue and green are often used as relatively distinct colours from skin tones. However, a coloured backdrop has several disadvantages. The scene cannot contain the same colour as the background as it would be removed in matting. The backdrop can also introduce a mapping of the hue on to to the scene from inter-reflections. Green-screens can prove most distinct from typical clothing, however green can produce unnatural looking skin where blue is more complementary to skin tones.

Non-coloured backdrops such as black, white or grey, can be used to avoid constraints on the colour of the scene. However, a scene can also have a similar appearance to a neutral backdrop. A white background for example will match white clothing or surface highlights that saturate a camera. Self-shadowing will produce dark regions that may be indistinguishable from a black or grey background. It is also more difficult to correctly identify shadowed background regions which could feasibly be the same colour as the scene. Here, a coloured backdrop provides a fixed chroma independent of shading variations to simplify background identification.

Relatively recently retro-reflective backdrops, such as Truematte, have been developed to provide an evenly lit coloured background. Screens are made from a fabric that is woven with glass beads to provide a retroreflective surface. An LED ring is placed around a camera lens to illuminate the fabric and light is reflected directly back to the camera to give a coloured background. One advantage is that relatively low light levels can be used to illuminate the backdrop reducing the colour spill onto the foreground, however the material has a relatively higher cost.

Different backdrops used in two studios are shown in Figure 1. A neutral backdrop is used in Figure 1(a) to allow for different coloured clothing unaffected by inter-reflections from a background colour and a blue backdrop is used in Figure 1(b) to ensure high-quality foreground mattes. 


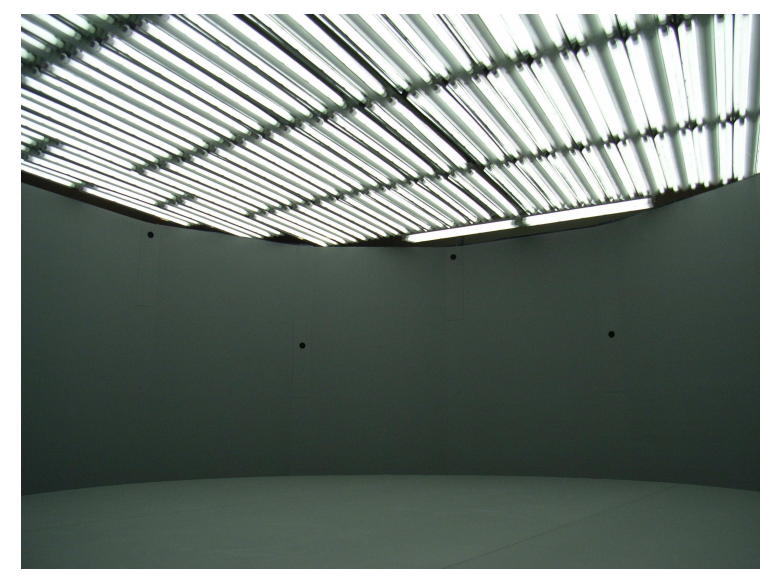

(a) Kyoto University [3]

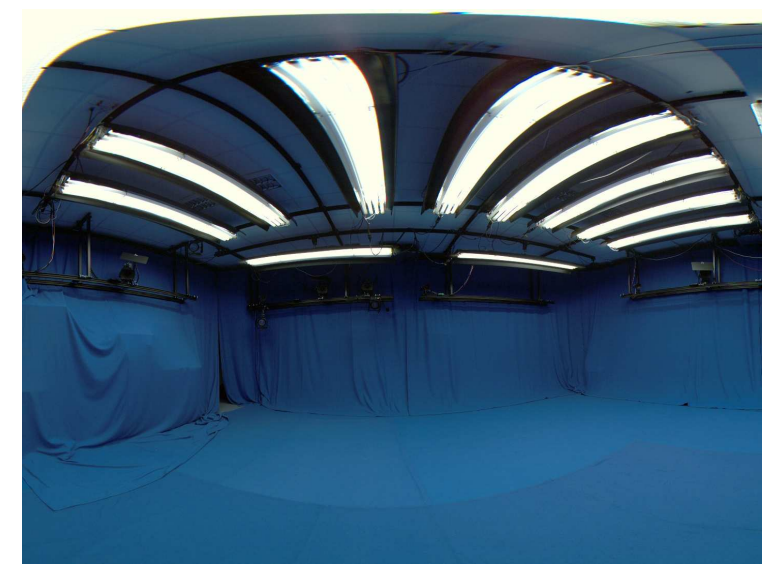

(b) University of Surrey [2]

Fig. 1. Prototype 3D Production Studios: (a) $6 \mathrm{~m}$ diameter, $2.2 \mathrm{~m}$ high cylindrical studio with 15 XGA-1CCD cameras using a neutral backdrop with overhead lighting and (b) 8m diameter, 3m high square studio with 8 HD-3CCD cameras using a blue backdrop with overhead lighting.

\section{B. Illumination}

Lighting affects both the appearance of the foreground scene and the studio backdrop in the recorded multiple view video. Ambient lighting, rather than directional spot-lights, is desirable. Ambient lighting provides an even appearance for the foreground with less self-shadowing. Ambient lighting also reduces the shadows cast on the background from the foreground. If overhead lights are assumed, directional lighting can cast dark shadows on the floor that can be incorrectly extracted as foreground in matting.

Lighting also has an impact on the camera setup. The aperture of the lens controls the amount of light that reaches the camera. A small aperture setting results in a larger depth-of-field, the range over which the scene is in focus. However there is a trade-off as a small aperture requires a slower shutter speed to increase the amount of light, whereas a fast shutter speed is required to reduce motion blur. It is therefore a fundamental requirement to ensure a large amount of incident illumination in the scene to allow a small aperture for greater depth-of-field and a fast shutter speed to reduce motion blur.

Achieving an even ambient illumination is a difficult task. With high lighting levels, cameras must be directed away from the lights to prevent saturation and blooming. A typical studio configuration uses cameras positioned overhead to surround the scene and overhead lighting out of the field-of-view for the cameras. One solution to the ambient lighting is to line the ceiling with fluorescent light tubes, as illustrated in Figure 1. It is important to ensure that the lights have a consistent colour spectrum to prevent 
uneven illumination from different areas of the ceiling and flickerless operation is required to ensure that the lighting level is consistent over a video sequence.

\section{Cameras}

Making the right choice of camera is crucial in designing a studio. There is a clear trade-off between the cost of the camera system and the number or resolution of the cameras required. At one end of the spectrum there is the high-cost camera equipment that would be used in a broadcast or film production scenario, see for example [2]. These systems use professional 3-CCD cameras and lenses that can typically capture uncompressed 24bpp colour resolution images to a dedicated capture server per camera. This high-cost solution can provide high resolution and high colour-depth images but has high-bandwidth requirements and the cost limits the number of cameras available for a given budget.

Digital machine vision cameras are often used as a relatively low cost solution, see for example [26]. There are three different types of cameras characterised according to their interfaces, IEEE1394 (FireWire or i.Link), GigE Vision, and CameraLink. The choice of camera is governed by the bandwidth and resolution as well as for example the cable length that can be used according to the size of the studio. The characteristic of each interface is summarised in Table II. It is a critical requirement that all cameras are synchronised to sample a dynamic scene over the same time period from each camera viewpoint, prohibiting the use of many consumer grade cameras.

IEEE1394 (FireWire or i.Link) is a serial bus with a data rate of $400 \mathrm{Mbps}$ or $800 \mathrm{Mbps}$. This gives a maximum video resolution of XGA or UXGA/HD for 1CCD 8bpp data at 30fps. The maximum metal cable length is $4.5 \mathrm{~m}$ with extensions provided by repeators. Optic fibre cable is also available with opticelectronic converters. Since this is a packet-based bus interface external trigger signals cannot be sent, although some cameras accept a "magic packet" which allows several cameras on different buses to synchronize. GigE Vision is another specification for a packet-based bus interface which utilizes gigabit ethernet as the physical layer. This system can transfer data at $1 \mathrm{Gbps}$ giving a maximum resolution of UXGA/HD video at $30 \mathrm{fps}$ for 1CCD 8bpp data. The cable length is up to $100 \mathrm{~m}$. The CameraLink interface connects cameras directly to enable real-time control including trigger signals for synchronisation. Data can be transferred at UXGA resolution for $3 \mathrm{CCD}$ video at 30fps. The cable length can be up to $10 \mathrm{~m}$ depending on the desired bitrate and optic fibre cables with an optic-electronic converter can be used to extend the cable length. It is also possible to provide power to cameras if used with PoCL (power over CameraLink) cables. High quality video images are required both to simplify tasks such as foreground matting and image matching for geometric scene reconstruction, as well as to provide the highest surface 


\begin{tabular}{|c|c|c|c|}
\hline & IEEE1394 & GigE vision & CameraLink \\
\hline Bandwidth & $\begin{array}{c}0.4 \mathrm{Gbps}(\mathrm{a}) \\
0.8 \mathrm{Gbps}(\mathrm{b})\end{array}$ & 1.0Gbps & $\begin{array}{c}\text { 2.04Gbps(base) } \\
\text { 4.08Gbps(medium) } \\
5.44 \mathrm{Gbps}(\mathrm{full})\end{array}$ \\
\hline $\begin{array}{c}\text { Resolution } \\
\text { at 30fps }\end{array}$ & $\begin{array}{c}\text { XGA 8bpp(a) } \\
\text { UXGA 8bpp(b) }\end{array}$ & UXGA 8bpp & UXGA 24bpp \\
\hline $\begin{array}{c}\text { Cable length } \\
\text { with repeaters }\end{array}$ & $\begin{array}{c}4.5 \mathrm{~m} \\
72 \mathrm{~m}\end{array}$ & $\begin{array}{c}100 \mathrm{~m} \\
\text { unlimited }\end{array}$ & $\begin{array}{c}10 \mathrm{~m} \\
\text { unlimited }\end{array}$ \\
\hline Synch & N/A & N/A & Available \\
\hline Grabber & Not required & Not required & Required \\
\hline Daisy chain & OK & OK & N/A \\
\hline
\end{tabular}

TABLE II

MACHINE Vision CAMERA INTERFACES

sampling to synthesise virtual views. Progressive scan (or frame mode) cameras provide a higher vertical image resolution for dynamic scenes compared to interlaced (field mode) cameras where alternating fields are sampled. Three-CCD cameras provide a higher colour depth compared to single CCD cameras. A 1-CCD camera requires a Bayer-filter to sample different colours at each pixel giving an 8bpp raw image, the colours are then demosaiced to produce a complete 24bpp colour image. Colour bleeding will occur particularly at distinct colour boundaries such as a blue-screen backdrop and the mosaic pattern can remain apparent in the image reducing the effective spatial sampling rate.

\section{Camera setup}

An intuitive way to record a 3D capture space with multiple cameras would be to place the cameras at an equal distance apart so that the target, typically located in the centre of the studio, can be uniformly viewed regardless of its pose or direction. Systems typically use a fixed set of cameras focused on a restricted volume, although real-time tracking can be used to provide an extended capture space [39].

Camera placement is governed by the required reconstruction accuracy and the required sampling resolution over the target capture volume. Reconstruction accuracy is dependent on the visual cues used for reconstruction. Foreground silhouettes define the outline boundary of an object projected to a 2D image. Shape-from-silhouette performs an intersection of these boundaries that provides an upperbound on the true shape of the object that is only guaranteed to be accurate along the object rims 
that generate the silhouette images. Reconstruction accuracy is dependent on sampling sufficient rims to approximate the underlying scene and typically requires a large number of evenly spaced camera views. Stereo correspondence on the other hand can recover dense surface geometry by matching appearance between viewpoints. Matching is based on maximising the correlation between corresponding patches in camera images. At short baselines, appearance will be similar increasing the likelihood that a good match can be recovered, however the accuracy in recovering depth is reduced as a small error in image matching can lead to large errors in depth. Conversely with large baselines, appearance will vary between image patches reducing the chance of a good match, however the reconstruction accuracy is increased due to a reduced depth ambiguity. Cameras should ideally be placed to maximise the accuracy from these complementary shape cues in reconstruction.

For a fixed arrangement of cameras the sampling resolution will define both the reconstruction accuracy that can be achieved and the sampling of surface appearance to synthesise new views. As the surface sampling rate increases, finer resolution matches will be derived which will reduce the reconstruction error. Sampling is effectively governed by the resolution of the output image, defined by the number of sampling elements on the camera and any resampling to construct the output image, as well as camera zoom, the focal-length of the lens controlling the field-of-view in the scene that is imaged. A higher image resolution and a restricted field of view will increase the surface sampling rate. However, a restricted field-of-view will require a greater number of cameras to cover the entire scene.

\section{E. Calibration}

Camera calibration is a fundamental step in capturing 3D digital media that directly affects both the accuracy of 3D shape capture and the quality of visual synthesis. For studio production where cameras may be reconfigured many times in one day and the recording time for paid performers is critical, simple and quick methods of calibration are of key practical importance.

Geometric calibration must define the intrinsic (focal length, centre-of-projection, radial distortion) and extrinsic (pose, orientation) camera parameters. Techniques are characterised by the available input, namely point correspondences which can be between 2D images or between a 2D image and 3D space. Popular approaches include a 1-step calibration process or a 2-step process. In a 1-step approach, both the intrinsic and extrinsic parameters are calibrated with a fixed 3D object, using for example Tsai's algorithm [40]. A 2-step approach recovers intrinsic parameters first using a known object such as a planar chart [41] and then derives extrinsics using 2D to 2D point correspondences between images [42]. A clear prerequisite is that points should cover the entire studio to ensure accurate reconstruction for 
the 3D capture volume. Point-based [43] and wand-based [44] techniques provide a flexible method to calibrate opposing or non-overlapping cameras where a chart for example would not be simultaneously visible from all camera viewpoints.

Photometric calibration is required to balance the sampled appearance in different cameras. Within a camera vignetting distortion can occur, where image brightness decreases with distance from the image centre as a result of shading in the camera lens. In practise compensation for vignetting is not required unless a wide aperture is used. Differences in appearance will however occur between cameras due to different colour responses. A simple colour calibration step matches the white-point, together with the black and white levels for the scene in each camera. The white point defines the relative proportion of red, green and blue components defining the colour white and the levels serve to match the dynamic range for the scene. More accurate calibration requires matching of the individual colour response curves across different cameras, for example by adjusting the offset and gain to match a linear response with gamma correction switched off, or by calibrating the individual colour response curves [45].

\section{F. Summary}

Studio design choices are governed by the type of scenes that will be recorded and the state-ofthe-art techniques for a given budget. The backdrop is chosen to give the maximum contrast with a foreground scene for accurate foreground matting with the minimum impact of the content of the scene. The maximum amount of consistent ambient illumination is required that can be placed out of view of the camera acquisition system. Camera calibration should be as accurate as can be achieved using state-of-the-art techniques. Design decisions are therefore reduced to the physical arrangement and setup of the multiple camera system for 3D production. The cost for the choice on the quality and number of cameras will then in turn influence the design of the lighting and backdrop for a fixed studio budget. Supply of studio equipment is territory dependent, for example chroma-key backdrop and studio rigging equipment can be obtained from broadcast suppliers, retro-reflective material (reflecmedia.com), lighting (cirrolite.com, kino-flo.com), cameras (Sony, ptgrey.com), multi-camera storage (Sony, ioindustries.com, DVS) and complete capture solutions (fourdviews.jexiste.fr).

\section{GROUND TRUTH EVALUATION}

A quantitative evaluation is now presented using a ground-truth synthetic data-set to address design decisions in a 3D production studio. Experiments are performed to illustrate the effect of geometric accuracy in answering the following questions: How many cameras are required? Where should cameras 


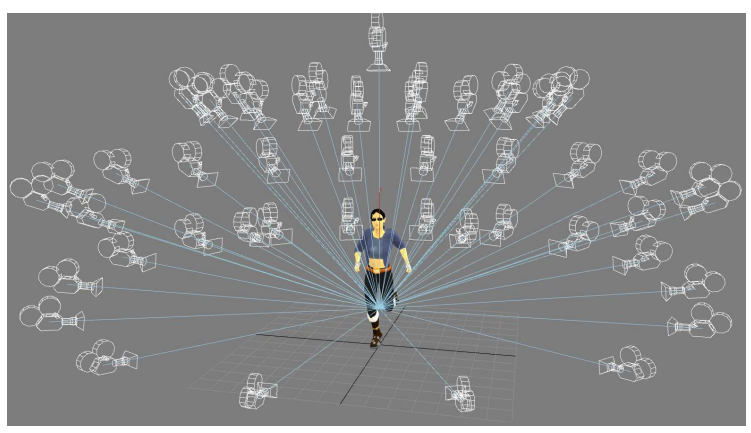

Fig. 2. Simulated cameras in a $8 \mathrm{~m} \times 8 \mathrm{~m}$ studio with a ceiling height of $4 \mathrm{~m}, 49$ cameras are configured in 3 rings of 16 cameras with one additional camera placed overhead.

be placed? What camera settings are required? and What is the influence of the target application of 3D production for visual synthesis?

\section{A. Synthetic data}

Multiple view video data is simulated with ground truth scene geometry. A single fixed size studio is used corresponding to a floor area of $8 \mathrm{~m} \times 8 \mathrm{~m}$ and a ceiling height of $4 \mathrm{~m}$. Cameras are placed in the simulated studio in a series of rings as shown in Figure 2. Where all cameras are used this corresponds to three rings each with 16 cameras and one camera is placed overhead giving a total of 49 cameras. The full camera configuration reflects the relatively large number of cameras used in the Virtualized Reality project [28], Table I. XGA resolution camera images $(1024 \times 768)$ are used with the subject forming approximately $55 \%$ the image height to give a capture space of $4 \mathrm{~m} \times 4 \mathrm{~m}$. Cameras are repositioned to simulate different baselines, they are excluded to simulate different camera sets and the image size is varied to simulate different capture resolutions.

A synthetic data-set is constructed and rendered using the 3D modelling and animation package 3ds Max. A human model is adopted consisting of 20k vertices and 40k polygons as shown in Figure 3(a). The model is textured from real images to simulate real video as shown in Figure 3(b) and the model consists of several layers of clothing to simulate occlusions in the images from both the gross articulation of the body and cloth movement. The model is animated using motion capture data. A single running sequence is used consisting of 21 frames of motion where the model is constrained to remain at the centre of the studio.

A real-world data-set is constructed using a public domain free-viewpoint video database from the University of Surrey [2]. The database consists of 8 motion sequences and 2220 frames of 8 camera HD 

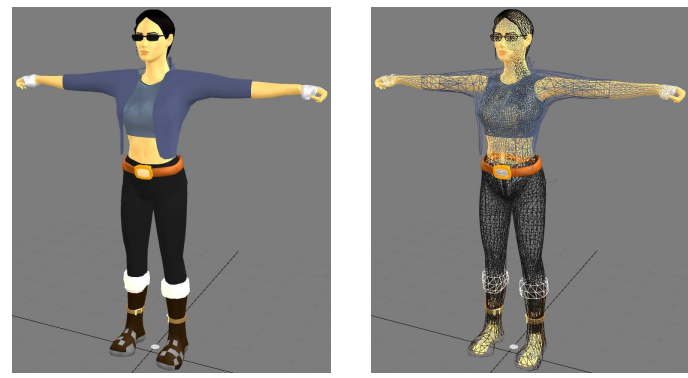

(a) Human model
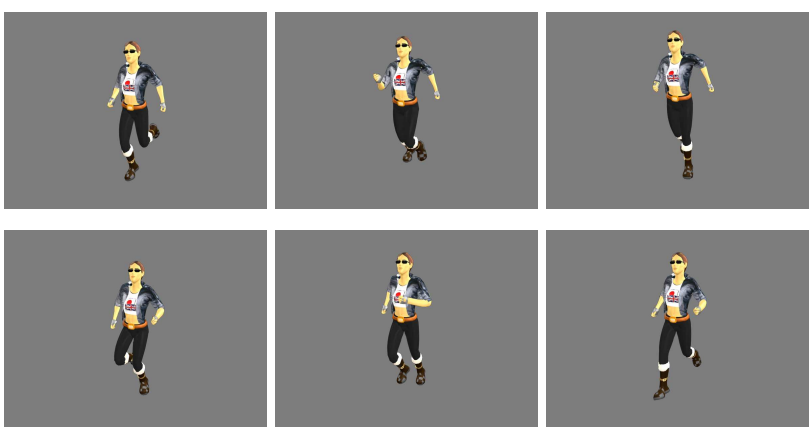

(b) Rendered animation sequence

Fig. 3. Synthetic model in 3ds Max animated using motion capture data and rendered using cloth plus hair texture to simulate real video images.

video data together with the geometry reconstructed using a global surface optimisation technique. The reconstruction technique [2] is compared to the state-of-the-art courtesy of the Middlebury Multi-View Stereo Evaluation project (vision.middlebury.edu/mview) [10]. Multiple-view video is synthesised for 21 frames randomly selected from the database using the public domain free-viewpoint video renderer (sourceforge.net/projects/fvvr). The renderer is modified for view-independent visualisation to ensure a consistent surface appearance between synthetic cameras. The data-set provides ground-truth geometry across real-world sequences with variations in motion complexity and real-world video texture for a human performer wearing everyday clothing as shown in Figure 4.
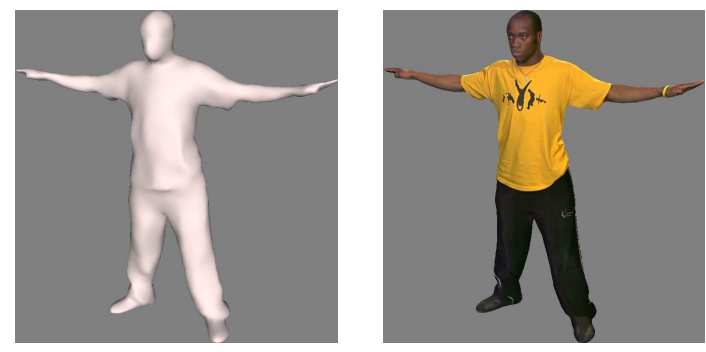

(a) Human model
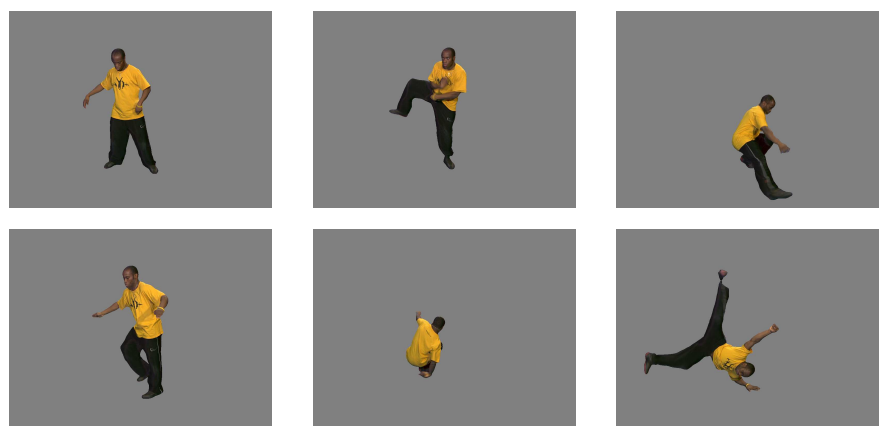

(b) Rendered animation sequence

Fig. 4. Surface model rendered using the Free-viewpoint Video Renderer (sourceforge.net/projects/fvvr) to simulate multiple view video data with ground truth geometry for real-world sequences of a person performing in a studio.

The geometric models in the data-sets are formally defined as a triangulated surface mesh $M$, repre- 


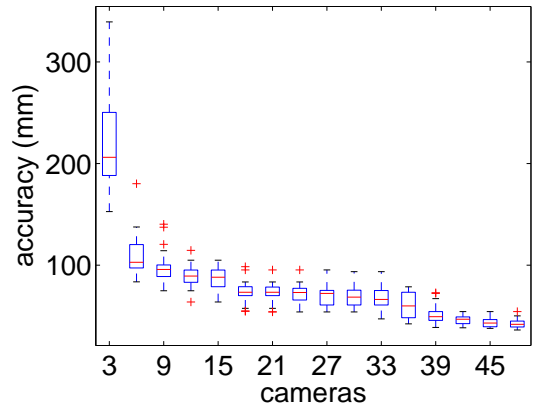

(a) Accuracy vs. cameras (Synthetic)

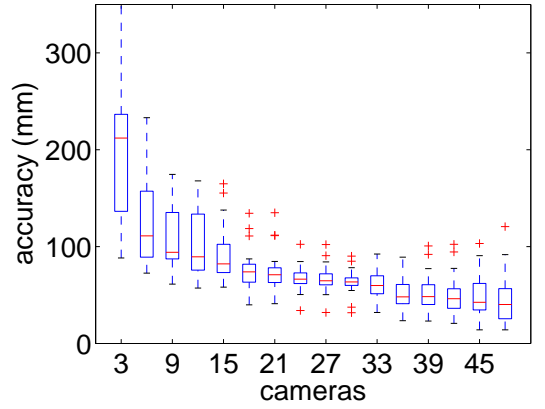

(b) Accuracy vs. cameras (Real)

Fig. 5. Reconstruction accuracy for shape from silhouette with the number of cameras used in reconstruction showing a box and whisker plot with the median, inter-quartile range and outliers greater than $\times 1.5$ the range.

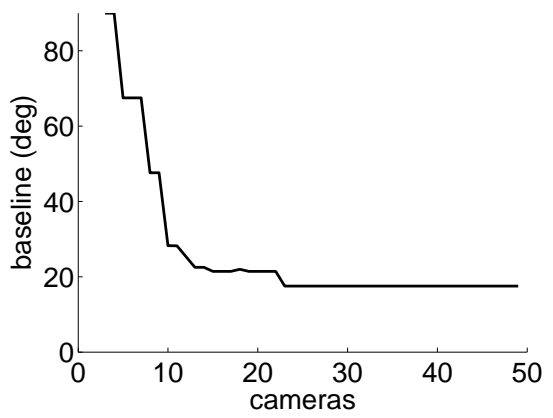

Fig. 6. Median camera baseline for the camera sets used to evaluate reconstruction accuracy for shape from silhouette.

sented by $(V, K)$ where $V$ is a finite set of vertex positions in $\mathbb{R}^{3}$ and $K$ is a simplicial complex defining the vertices $\{i\} \in K$, edges $\{i, j\} \in K$, and faces $\{i, j, k\} \in K$. For each frame corresponding to a time point $t$, a new mesh is defined, $M_{t}=\left(V_{t}, K_{t}\right)$. The rendered camera images are denoted by $I_{c, t}$ for camera $c$ at frame $t$ and the projection matrix $P_{c}$ is known. The data-sets used in the evaluation are denoted Synthetic and Real.

\section{B. Shape from Silhouette}

The choice for the number of cameras to achieve good reconstruction accuracy from silhouettes is evaluated by varying the number of camera views used in the multiple view video sequence. Cameras are selected to maximise the intersection angle between the cameras to provide a relatively even sampling of surface rims on the underlying scene. The minimum configuration considered is 3 orthogonal views with 
a front, top and side camera. Cameras are then iteratively added to maximise the angle subtended in the camera set. For each reconstructed mesh $M_{t}^{\prime}$ and each camera configuration the distance is computed to the ground truth surface $H\left(M_{t}^{\prime}, M_{t}\right)$. Here a one-sided Hausdorff distance $H$ is computed [46] to give the maximum reconstruction error from the reconstructed to ground-truth surface.

The accuracy for shape from silhouette is shown in Figure 5. With a limited set of cameras the reconstruction error is large. In the range 18 to 33 cameras the maximum reconstruction error is relatively stable at approximately $75 \mathrm{~mm}$ and the error only reduces below $50 \mathrm{~mm}$ with 42 or more cameras. It should be noted that certain classes of concavities are occluded in silhouette images and the error will not reduce to zero. Figure 6 shows the angle subtended between cameras for the camera sets used. Here it can be seen that the change in angle is highly non-linear. With a small number of cameras each additional camera causes a large change in angle, whereas with a larger camera set the change in angle is significantly reduced as cameras are added.

Conclusion 1. As the number of cameras used in reconstruction is increased, the accuracy in shape from silhouette increases. The angle subtended between camera views stabilises with 16 cameras and the corresponding reconstruction error changes relatively little without requiring significantly more cameras.

\section{Stereo Baseline}

The choice of camera baseline in stereo matching is evaluated by assessing the reconstruction accuracy for a single pair of cameras with a variable baseline. Matching is performed through conventional areabased image matching using a zero-mean normalised cross-correlation score (ZNCC) [47]. For a mesh sequence $M_{t}$, sample points are taken by randomly sampling the triangles $\{i, j, k\} \in K_{t}$ up to a fixed sampling rate of $1 \mathrm{sample} / \mathrm{cm}^{2}$. Image correspondence can fail at occlusion boundaries where there is a discontinuous change in appearance or regions of low image texture where there is insufficient image variation to match image patches. All sample points that are occluded in the cameras are culled by testing for occluding polygons in the projection to a camera viewpoint. A conservative visibility test is performed against an inflated mesh such that points near discontinuities become occluded. Image variation is tested by thresholding the standard deviation in pixel intensities at the projected sample location $\underline{u}_{c} \in I_{c}$ over the window size $W$ used for area-based stereo correlation as defined in Equation 1. Without loss of generality a fixed threshold $\sigma=10$ is adopted such that areas of uniform surface appearance for the model are explicitly excluded. 


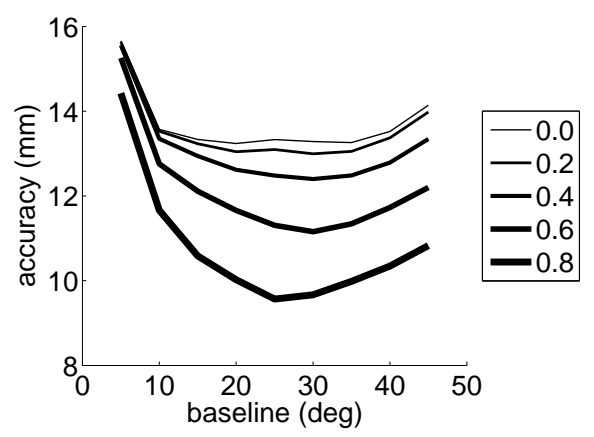

(a) Accuracy vs. baseline (Synthetic Data)

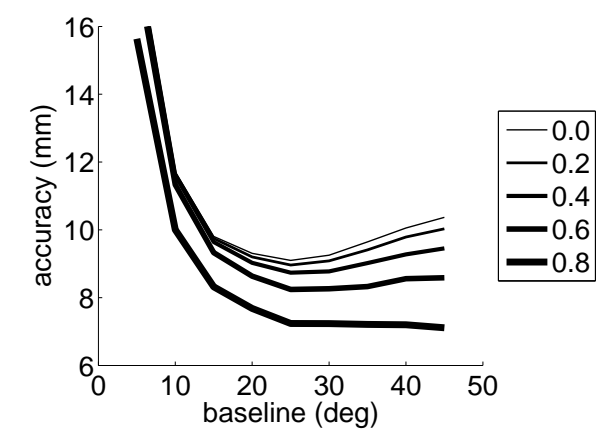

(c) Accuracy vs. baseline (Real Data)

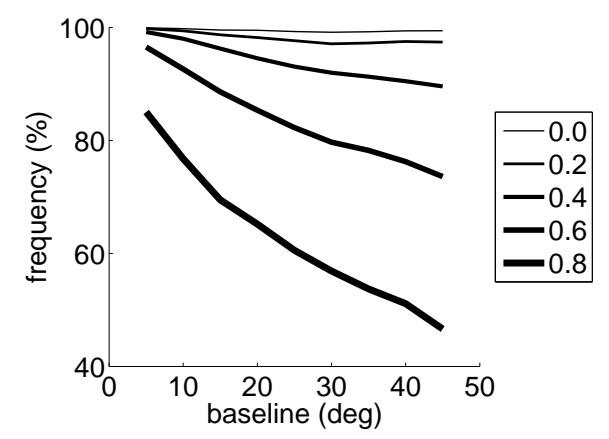

(b) Frequency vs. baseline (Synthetic Data)

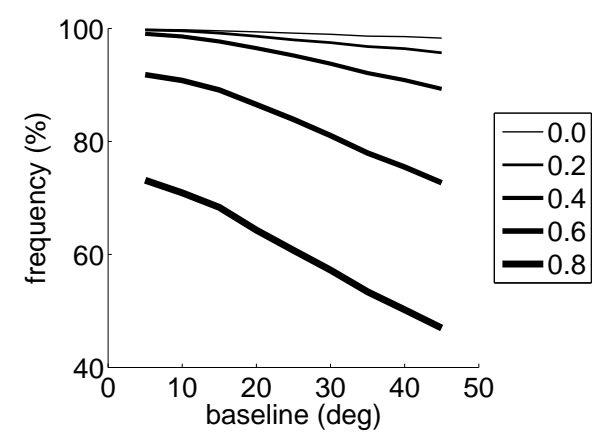

(d) Frequency vs. baseline (Real Data)

Fig. 7. Stereo matching performance with camera baseline showing (a,c) median reconstruction accuracy and (b,d) proportion of surface points, when considering only points with a correlation score greater than a threshold $\mathrm{C}$ in the range $(0.0$ to 0.8$)$.

$$
\sqrt{ } \sum_{\underline{w}}\left(I_{c}(\underline{u}+\underline{w})-\bar{I}_{c}(\underline{u})\right)^{2}>\sigma
$$

Given the visible set of samples considered for stereo matching $\underline{s} \in S_{t}$ a search is performed for the best stereo correlation between views. The projection of a sample point is fixed in one camera image, $P_{1} \underline{s}$, and a search is performed for the matching point in the second image, $P_{2} \underline{s}^{\prime}$ corresponding to the estimated surface position $\underline{s}^{\prime}$. The search is restricted to the epipolar line, the projection of a ray that connects the centre of the first camera and the known position $\underline{s}$ to the image plane, by rectifying the camera images [48]. Correspondence is defined where the correlation is maximised and a sub-pixel match is extracted by fitting a quadratic spline to the correlation scores adjacent to the maxima [49]. The estimated surface point $\underline{s}^{\prime}$ is recovered by triangulating the matched image positions and the geometric error $e=\left\|\underline{s}-\underline{s}^{\prime}\right\|$ together with the correlation score $c$ is recorded.

Stereo matches would only be considered either where there is local support for a match through a 
regularised search for correspondence or the correlation score is sufficiently high to warrant an acceptable match. The results are shown in Figure 7 against the threshold $C$ for an acceptable match. Figure 7(a,c) shows the median reconstruction accuracy for all points where $c>C, C=\{0.0,0.2,0.4,0.6,0.8\}$. It would be expected that at a wide-baseline with a reduced depth ambiguity there is the potential for greater accuracy but with a lower correlation score. The results show that as the threshold $C$ is reduced, the minimum error point does indeed move towards a wider baseline. However the graph clearly shows that the greatest accuracy is achieved when considering only the highest scoring matches $C=0.8$ at a baseline of $25^{\circ}$ demonstrating the trade-off between finding correct matches at a short baseline and reducing the depth ambiguity at a wide-baseline. Figure 7(b,d) shows the proportion of sample points that achieve the correlation threshold. Here the trade-off can be seen between using only a high threshold to improve reconstruction accuracy and using a low threshold to achieve dense surface reconstruction.

Conclusion 2. Optimal camera placement for stereo surface reconstruction follows the trade-off between accuracy in matching and depth ambiguity with baseline. The optimal baseline for the standard stereo matching technique adopted is $25^{\circ}$. For a planar camera ring this corresponds to 14 cameras or more in a non-planar configuration. Accuracy will be limited here by the assumption of fronto-parallel image patches and greater accuracy may be achieved by modelling the change in appearance between viewpoints.

\section{Visibility Requirements}

Camera placement is now evaluated in terms of surface visibility for a single camera ring. This camera setup is often used in the systems shown in Table I as it provides the maximum coverage from a limited set of cameras. Surface reconstruction ideally requires the coverage of all surface regions in multiple camera views to recover depth from image matches. View synthesis also requires coverage of all surface regions to completely sample the surface appearance to generate new views. Visibility is determined using a ray-tracing test. A 3D ray is cast from a point to a camera and the scene is tested for occlusions along the ray. Surface visibility is defined for each triangle in the mesh, $\{i, j, k\} \in K$, as follows.

- A triangle is visible from a camera $c$ if the ray connecting the triangle centroid and camera centre lies within the viewing frustum of the camera and has only one intersection, the source triangle.

- A triangle is potentially visible if a ray exists that connects the triangle centroid and infinity, with only one intersection corresponding to the source triangle.

Visibility is quantified as the relative proportion of visible surface area that is observed in a set of camera images, the ratio of the area for the visible set to the area of the potentially visible set of triangles. 


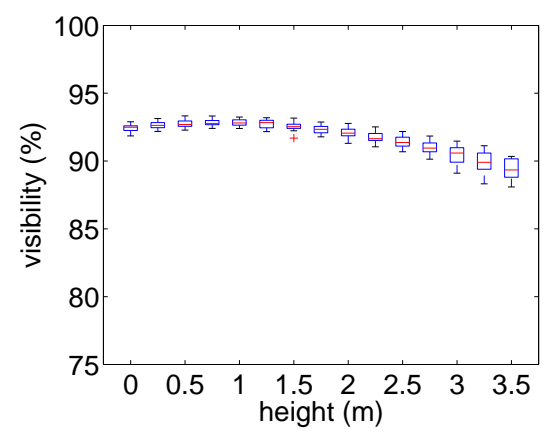

(a) Unconstrained visibility (Synthetic Data)

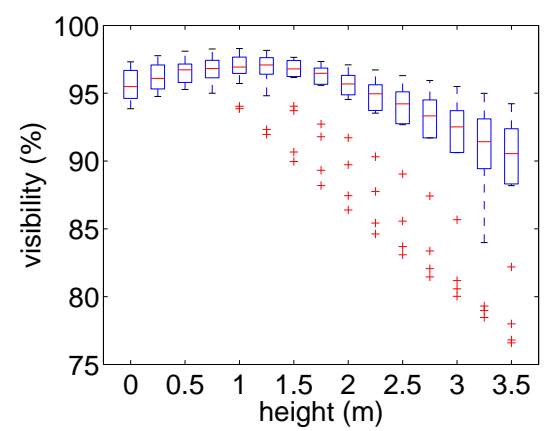

(c) Unconstrained visibility (Real Data)

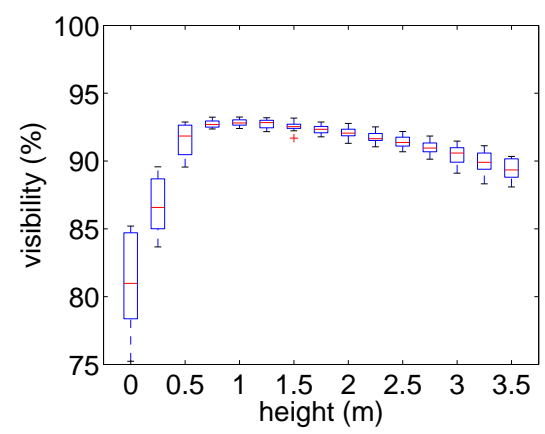

(b) Constrained visibility (Synthetic Data)

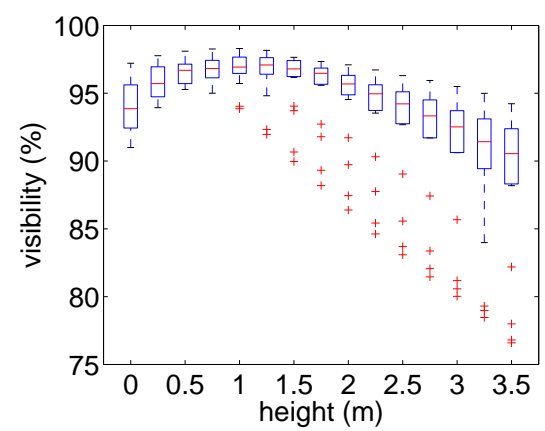

(d) Constrained visibility (Real Data)

Fig. 8. Synthetic data-set: Percentage surface visibility in a ring of 16 cameras as a function of camera height where (a,c) cameras are unconstrained and $(b, d)$ cameras are constrained such that overhead lighting is not in view.

A single ring with 16 cameras is used and the height of the ring is adjusted to evaluate the change in the surface visibility in the cameras. Two cases are considered, an unconstrained camera orientation where the height of the camera ring is moved from an overhead position to the floor and a constrained orientation where the camera viewpoint is automatically updated to exclude an $8 \mathrm{~m} \times 8 \mathrm{~m}$ ceiling area at $4 \mathrm{~m}$ high corresponding to a large area light source for ambient illumination.

Figure 8 shows the change in surface visibility as a function of camera height for the motion sequence. Visibility is maximised at a height of approximately $1 \mathrm{~m}$ in Figure 8(a,b,c,d), where both up and downward facing triangles are most likely to be visible in a camera. With a constrained camera viewpoint in Figure 8(b), it can be seen that surface visibility becomes markedly reduced below this height. At a low height, the upper part of the head in the synthetic data-set is excluded from the camera viewpoint as it would overlap with the ceiling area in the camera field of view.

Conclusion 3. Maximum surface visibility is achieved by placing cameras at the mid-height of the body, $1 \mathrm{~m}$ for the motion considered here. If cameras are placed overhead to exclude ceiling lights and 


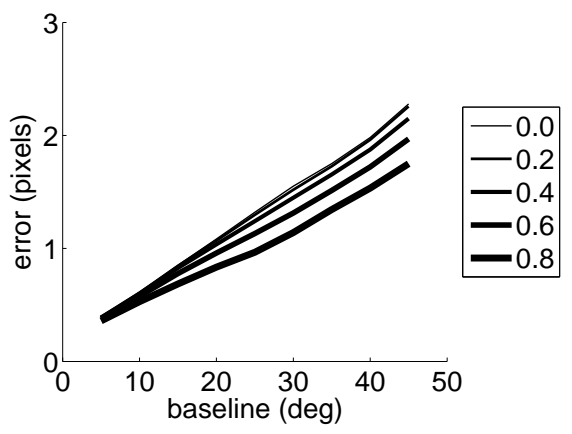

(a) Error vs. baseline (Synthetic Data)

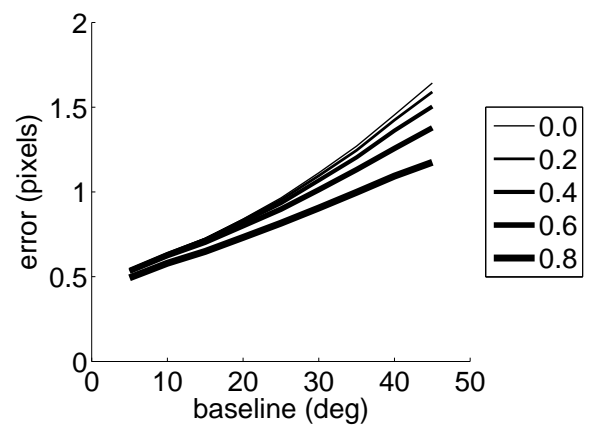

(c) Error vs. baseline (Real Data)

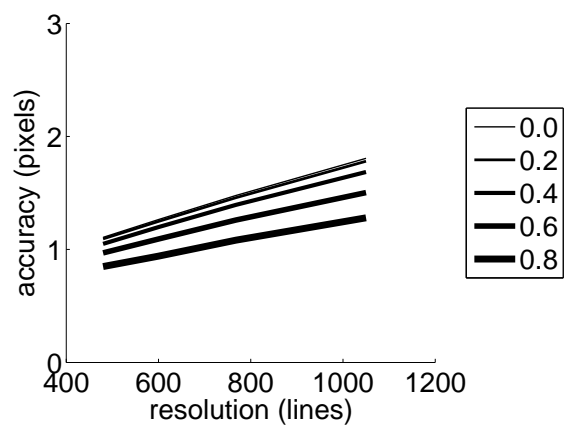

(b) Error vs. resolution (Synthetic Data)

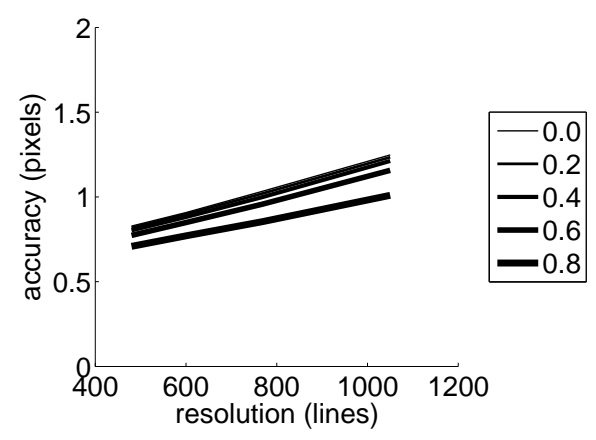

(d) Error vs. resolution (Real Data)

Fig. 9. Stereo matching performance shown in terms of the image plane matching error against (a,c) the camera baseline for XGA resolution images and (b,d) image resolution for a fixed baseline of $25^{\circ}$. Graphs show the median error for points with a correlation score greater than a threshold in the range (0.0 to 0.8$)$.

opposite facing cameras, then cameras have to be positioned above the maximum height of the recorded motion. The change in visibility with height is relatively small and additional cameras are required at a low level to complete the recovery of surface appearance at the cost of requiring improved background subtraction to handle the inclusion of camera equipment in the field of view of the overhead cameras.

\section{E. Camera Resolution and Zoom}

Stereo matching is now evaluated in terms of the pixel error in matching two images to determine the effect of image resolution and focal-length on geometric accuracy. In Section IV-C, performance was assessed by searching for stereo matches between two camera views. Figure 9(a) now shows the results for the experiments in Figure 7(a,c) in terms of image matching accuracy. Figure 9(a,c) demonstrates the ambiguity in matching images, at a wider baseline the variation in image appearance increases reducing the matching accuracy. 
Matching accuracy is evaluated with image resolution to simulate either a corresponding change in camera sampling rate, or an increase in focal-length where only a fixed window in the synthetic image would actually be used. Figure 9(b,d) shows the median accuracy for resolutions of VGA (480lines), SVGA (600lines), XGA (768lines) and SXGA+(1050lines) with a fixed aspect ratio of 4:3 and a fixed camera baseline of $25^{\circ}$. A stereo window size of $9 \times 9,11 \times 11,13 \times 13,17 \times 17$ is adopted respectively to capture a consistent surface area in the images. This range of resolutions corresponds to the camera systems used in the literature, Table I. Figure 9(b,d) demonstrates that matching accuracy decreases only slightly with an increase in camera resolution for both the synthetic and real-world data-sets.

For an estimated image matching accuracy it is possible to formulate the expected geometric reconstruction error. For a fixed camera baseline defined by the angle between the camera viewing directions $\theta$, at a subject distance of $D(\mathrm{~mm})$, a focal-length of $f(\mathrm{~mm})$ and an effective pixel size of $\mu(\mathrm{mm} / \mathrm{pixel})$ on the $\mathrm{CCD}$, the reconstruction error $\delta(\mathrm{mm})$ is governed by the matching accuracy $m$ (pixels) in Equation 2. As an order of magnitude calculation, the median matching accuracy is estimated as $m=1$ from Figure 9(b,d) for the optimal camera baseline $\theta=25^{\circ}$ providing a rule-of-thumb calculation for geometric error, $m / \sin (\theta)=2.4$. In practise matching accuracy can increase with greater appearance variation from real images, a higher appearance sampling rate such as a 12-bit colour depth, or it may decrease with a lower effective sampling rate due to a bayer pattern or greater CCD noise. The resulting reconstruction accuracy will also decrease in practise with camera calibration error. ${ }^{1}$

$$
\delta=\frac{m D \mu}{f \sin \theta}=2.4 \frac{D \mu}{f}
$$

This order of magnitude calculation gives some insight into achievable reconstruction accuracy. With a limited set of cameras, whole-body images are required to cover the desired capture volume. For the studio setup considered with $D=4 \mathrm{~m}$ and HD resolutions (1080lines), this equates to $\delta \approx 5 \mathrm{~mm}$ and at XGA resolution (768lines) $\delta \approx 10 \mathrm{~mm}$. This accuracy will not recover the detailed geometry in clothing wrinkles or shallow concavities such as the eyes, but is sufficient to recover gross cloth and body shape. To achieve a greater reconstruction accuracy the focal-length $f$ must be increased. However, it is important to note that this gives a restricted field of view requiring additional cameras to provide complete scene coverage and that an increase in focal-length comes with a decrease in the depth of field in the scene.

\footnotetext{
${ }^{1}$ At the time of writing the best case accuracy for the public domain multi-view data set presented in [10] indicates a value of 2.1 in Equation 2 for $90 \%$ of the reconstructed surface. The data-set uses 16 camera views recorded from a single (hence perfectly matched) VGA camera where the variation in appearance between views is modelled.
} 


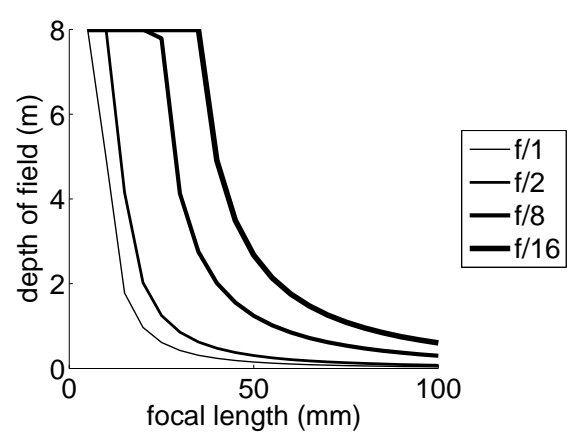

Fig. 10. Depth of Field values calculated for a simple optical model [50] at a subject distance of $4 \mathrm{~m}$ with a circle of confusion of $12 \mu \mathrm{m}$.

Depth of field (DOF) is defined by the minimum and maximum distance from a camera that the scene appears to remain sharp. In designing a studio, the parameters controlling depth of field are highly constrained. The camera to subject distance is governed by the physical size of the capture space. The aperture (f-stop) is defined to achieve the desired dynamic range in the images under the maximum studio illumination that can be achieved. Focal-length is governed by the desired sampling rate in the scene given sufficiently available cameras or the desired field of view to cover a specific capture volume given a restricted number of cameras. Figure 10 illustrates the influence of focal-length and f-stop on DOF under a simple optical model [50].

Case-study: At the University of Surrey, HD 1080p images are used with $\mu=0.005 \mathrm{~mm}$, circle of confusion $12 \mu \mathrm{m}$ in a $4 \mathrm{~m}$ diameter studio. This requires a $10 \mathrm{~mm}$ lens for whole body images giving $\delta \approx 5 \mathrm{~mm}$ and a $47 \mathrm{~mm}$ lens for partial body images and $\delta \approx 1 \mathrm{~mm}$. With an aperture f/5 under 4000 lux illumination the DOF from Figure 10 is sufficient to cover the studio at $10 \mathrm{~mm}$ but is reduced to just $0.8 \mathrm{~m}$ with a $47 \mathrm{~mm}$ lens.

Conclusion 4. Geometric error can be estimated by a simple order of magnitude calculation. For whole-body images and current generation cameras the achievable accuracy is around 5-10mm which is insufficient to recover fine cloth wrinkles or the detailed geometry in the face. As camera zoom is increased to achieve a greater sampling rate, the reconstruction accuracy will increase however the depth of field in the scene will be reduced. Additional cameras would be required to not only provide a complete scene coverage, but multiple camera sets would be required focused at different subject distances such that the entire capture volume can be imaged in focus. 


\section{F. View synthesis}

Shape capture is now considered as the problem of recovering a sufficiently accurate shape to synthesise a new viewpoint without artefacts. Limited reconstruction accuracy has two effects in synthesis. Firstly the surface appearance can be inconsistent between cameras when the geometry is not correct and so the appearance must be estimated from the available samples. Secondly, the appearance may be consistent but incorrect giving an incorrect appearance when synthesising a new view. With a fixed surface appearance, the inconsistency between cameras can be minimised by recovering appearance from the camera images where the surface sampling rate is maximised [2]. However, a fixed appearance on incorrect geometry may not reproduce the motion parallax expected with a change in synthetic viewpoint. For example at a $10 \mathrm{~mm}$ reconstruction accuracy the concavities at the eyes will not be recovered. If appearance is sampled from a frontal camera where the highest sampling rate is achieved, as a virtual view moves to one side the eyes will appear to bulge. View-dependent rendering can overcome this limitation [19], providing the appearance of a concavity by rendering the change in appearance sampled in the camera images to the side. However, geometric error can result in different appearance samples between the camera images used in rendering causing in blurring and double exposure effects in view synthesis whereas the sharpest image appearance will be provided by a single fixed surface appearance [2].

For a fixed surface appearance, the reprojection error with motion parallax is considered. From Equation 2 , the reprojection error $m$ can be calculated at a given camera angle $\theta$ for a given reconstruction accuracy $\delta$. Figure 11 translates the reconstruction accuracy in Figure 7(a,c) with camera baseline to a reprojection error for a virtual viewpoint positioned at $90^{\circ}$. A minimum reprojection error around 2pixels occurs with the minimum reconstruction error at a $25^{\circ}$ camera baseline. To achieve sub-pixel accurate view-synthesis $m<1$ at $\theta=90^{\circ}$ with a fixed surface appearance, the synthesised image dimensions should be less than half that of the camera images.

For a view-dependent surface appearance, as the baseline between blended camera images increases so does the projection error between the images for a given geometric error. A virtual view-point will be consistent up to the reprojection error between the camera images that are blended. Under the assumption that matching accuracy $\hat{m}$ for a camera system with a baseline $\hat{\theta}$ is constant in Equation 2, then it is possible to define the maximum allowable angle $\theta_{\max }$ between viewpoints for a desired reprojection error $m_{\max }$ for a synthesised view at the same resolution as the camera system. For sub-pixel accuracy

$m_{\max }<1$ with $\hat{m}=1, \hat{\theta}=25^{\circ}$ then trivially $\theta_{\max }<25^{\circ}$. As the resolution of the synthesised view decreases with respect to the camera system the maximum angle between camera viewpoints will then 


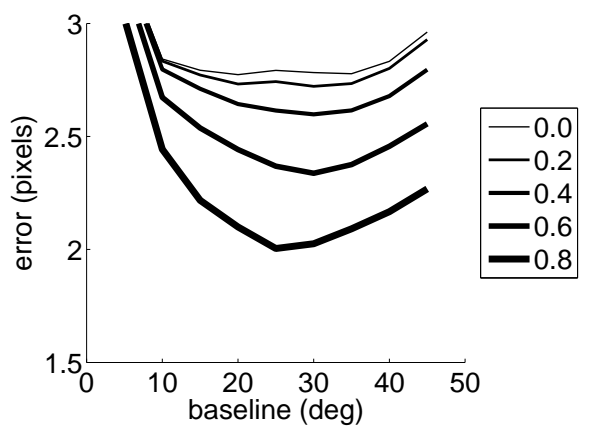

(c) $90^{\circ}$ error vs. baseline (Synthetic Data)

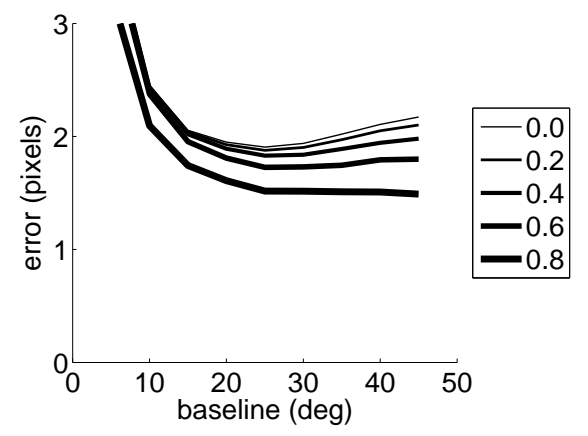

(d) $90^{\circ}$ error vs. baseline (Real Data)

Fig. 11. Reprojection error in a virtual camera with a $90^{\circ}$ view point for surface points reconstructed at different camera baselines where correlation exceeds a threshold in the range (0.0 to 0.8$)$.

increase.

Conclusion 5. With a fixed surface appearance sub-pixel reprojection errors in motion parallax will only be achieved by reducing the resolution in view synthesis compared to the captured image resolution. With a view-dependent appearance errors in motion parallax will be corrected and sub-pixel reprojection errors can be achieved at the captured image resolution with a camera baseline of $\theta<25^{\circ}$.

\section{A COMPARISON OF TWO STUDIOS}

Two prototype studios developed at the University of Surrey and Kyoto University are now described and a qualitative comparison of human performance capture is presented. Table III summarises and contrasts the components of the two studio systems.

\section{A. University of Surrey}

At the University of Surrey a low number of professional High-Definition (HD) 3CCD cameras are used to provide high quality appearance capture. Eight Thomson Viper cameras are equally spaced in a ring of $8 \mathrm{~m}$ diameter at a height of $2 \mathrm{~m}$ above the studio floor. An even ambient illumination of around 4000 lux is provided by an array of KinoFlo fluorescent tubes on the ceiling, with flickerless operation and a consistent colour spectrum. The cameras are positioned above the capture volume and directed downward to exclude the lighting from the field-of-view. The cameras have a wide $45^{\circ}$ baseline to provide $360^{\circ}$ coverage of a capture volume of $4 \times 4 \times 2 \mathrm{~m}$. A blue-screen backdrop is used for foreground segmentation as shown in Figure 1(b). Performances are captured in HD-SDI 20-bit 4:2:2 format with 


\begin{tabular}{lcc}
\hline & University of Surrey & Kyoto University \\
\hline $\begin{array}{l}\text { Backdrop } \\
\text { pros }\end{array}$ & Blue-screen & Grey-screen \\
cons & Simple foreground matting & Minimises coloured reflections \\
\hline Cameras & ICCD/HD/Broadcast & 1CCD/XGA/Machine vision \\
pros & High quality, high spatial sampling & Low cost, increased number of cameras \\
cons & High cost & Moderate quality \\
\hline Configuration & 8 camera, planar ring & 15 camera, non-planar \\
pros & $360^{\circ}$ horizontal coverage & Complex shadow detection, no grey clothing \\
cons & Limited camera set & $360{ }^{\circ}$ coverage, increased vertical coverage \\
\hline Lighting & Overhead fluorescents & Opposing cameras in field-of-view \\
pros & Even ambient illumination & Overhead fluorescents \\
cons & Some self-shadowing & Even ambient illumination \\
\hline
\end{tabular}

TABLE III

COMPARISON OF STUDIO COMPONENTS

$1920 \times 1080$ resolution at $25 \mathrm{~Hz}$ progressive scan. Synchronized video from all eight cameras are recorded uncompressed direct to disk with eight dedicated PC capture boxes using DVS HD capture cards. Shape reconstruction is performed using a global optimisation technique [2].

\section{B. Kyoto University}

At Kyoto University 15 machine vision IEEE1394a cameras are employed to capture intangible cultural assets. Two sets of 6 cameras are arranged in double rings of $6 \mathrm{~m}$ diameter at heights of $1.2 \mathrm{~m}$ and $2.2 \mathrm{~m}$, respectively. An extra camera on the studio wall is dedicated to capture the face of a target subject, and the remaining 2 cameras are placed on the ceiling. A uniform ambient illumination is provided by an array of TRUE-LITE (850L, 5500K) fluorescent tubes as shown in Figure 1(a). The cameras in the rings have a $30^{\circ}$ baseline and provide a $3 \times 3 \times 2 \mathrm{~m}$ capture volume. A grey background is adopted using grey-painted plywood panels to avoid inter-reflected colour. Performance is captured in the raw bayer format (8bit per pixel) with $1024 \times 768$ resolution at $30 \mathrm{~Hz}$ progressive scan. Cameras are synchronised using an external trigger (DCAM MODE_0) and raw uncompressed data (8bit XGA @ 25fps = 18.75M bytes/sec) is recorded direct to SCSI disk via IEEE 1394a using 15 dedicated PCs. Shape reconstruction is carried out by a surface optimization technique using a deformable mesh [3]. 


\section{Results for human performance capture}

Performance capture is now illustrated across the two studios. Two data-sets are considered, the first recorded at the University of Surrey with a street-dancer performing fast acrobatic motions wearing everyday clothing and the second recorded at Kyoto University for a Maiko wearing a brightly coloured Kimono performing a slow dance. A qualitative comparison is presented for both geometric reconstruction [2], [3] and view synthesis using view-dependent texturing [19] from the camera images.

Geometric reconstruction is performed using optimisation of the visual-hull surface [3] with an average computation time of $1 \mathrm{~min} /$ frame on an Intel(R) Xeon(TM) $3.6 \mathrm{GHz}$ CPU and global optimisation inside the volume defined by the visual-hull [2] with a computation time of $38 \mathrm{~min} /$ frame on an Intel(R) Xeon(TM) 3GHz CPU. The reconstructed surfaces for each technique are illustrated for the two data-sets in Figures 12(a) and 13(a). Surface optimisation provides a more efficient technique that is dependent on the quality of the initial surface. With a limited set of cameras and multiple self-occlusions in camera images phantom volumes can occur in the visual-hull. These structures will persist where visual cues such as colour variation or texture are limited. Global optimisation can produce a more accurate reconstruction at a higher computational cost.

Figures 12(b), 13(b) show view synthesis not aligned with the original camera viewpoints for the reconstructed geometry. Where geometric shape is exact, the surface appearance will be blended correctly across the camera images up to the calibration accuracy of the camera system. Geometric errors can lead to incorrect blending of sampled appearance and incorrect estimation of surface visibility in the camera images. For the fast motions in Figure 12 any blur arising from small scale geometric error is masked by the motion blur in the original camera images. However, gross geometric errors arising from phantom structures lead to incorrect samples of surface appearance from the images and incorrect structures becoming apparent in view synthesis. Geometric reconstruction should recover a sufficiently accurate scene representation for view synthesis in terms of both the rendered surface appearance and the rendered shape, the outline of the rendered scene.

\section{CONCLUSION}

Achieving high quality $3 \mathrm{D}$ reconstruction represents a significant challenge in a multiple camera production studio. The different design decisions in constructing a studio have been presented and two real studios have been compared. A ground truth evaluation for the impact of geometric reconstruction accuracy has been performed to provide a quantified insight into the effect of different choices governing 

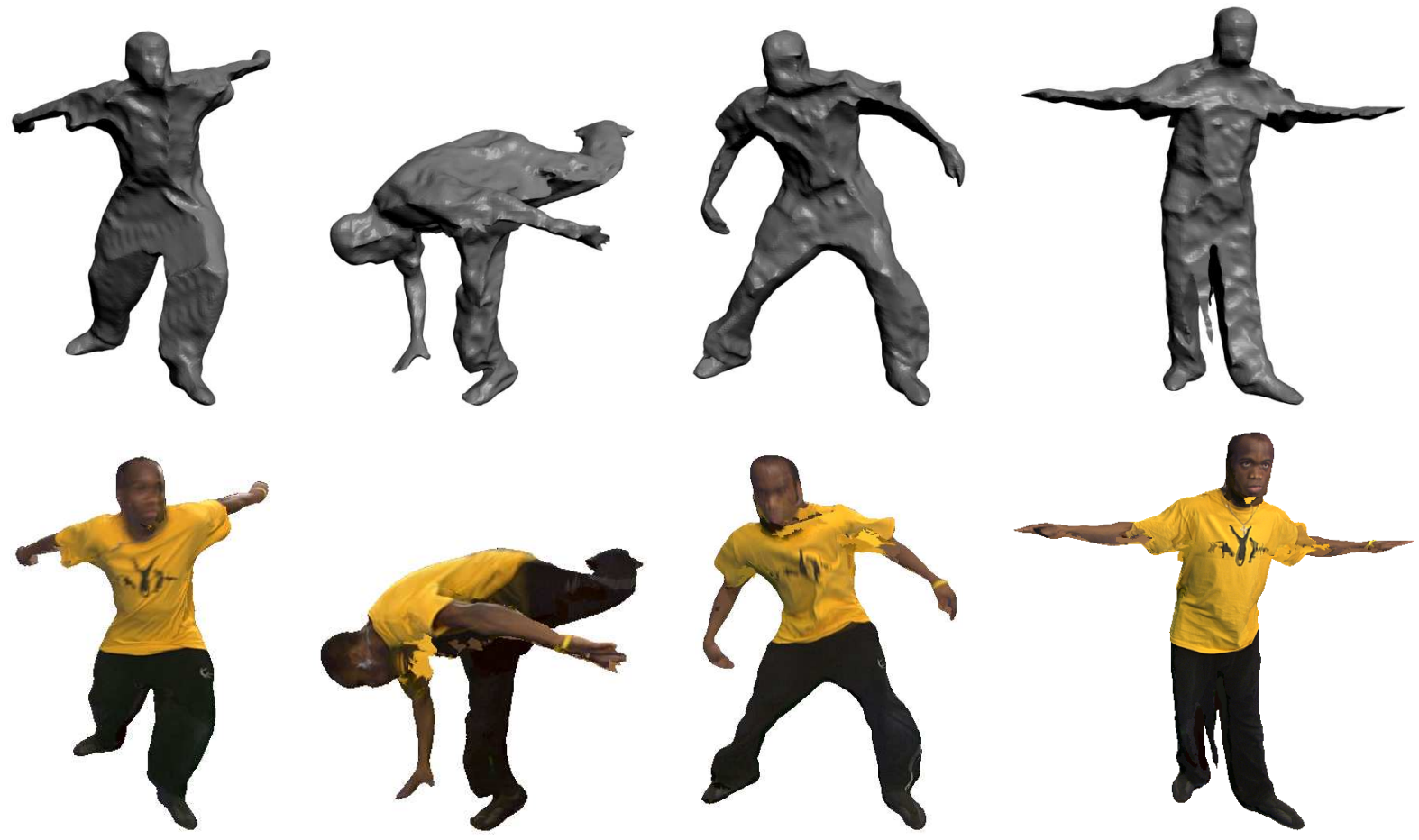

(a) Reconstruction using shape optimisation [3] and view-dependent rendering.
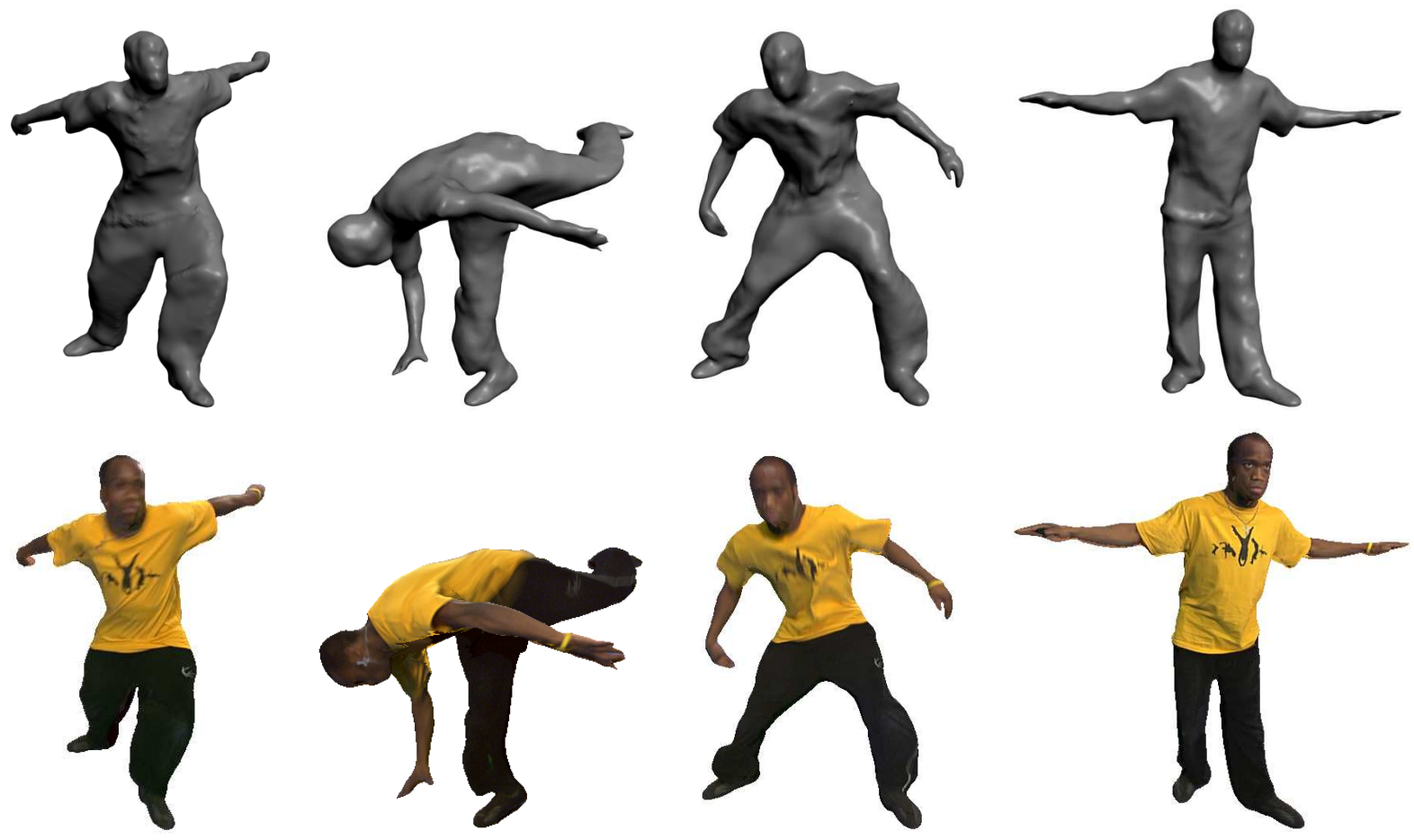

(b) Reconstruction using global optimisation [2] and view-dependent rendering.

Fig. 12. Comparison for the publicly available [2] street-dance sequence recorded at the University of Surrey. Note that the fast motions lead to motion blur in view synthesis. 

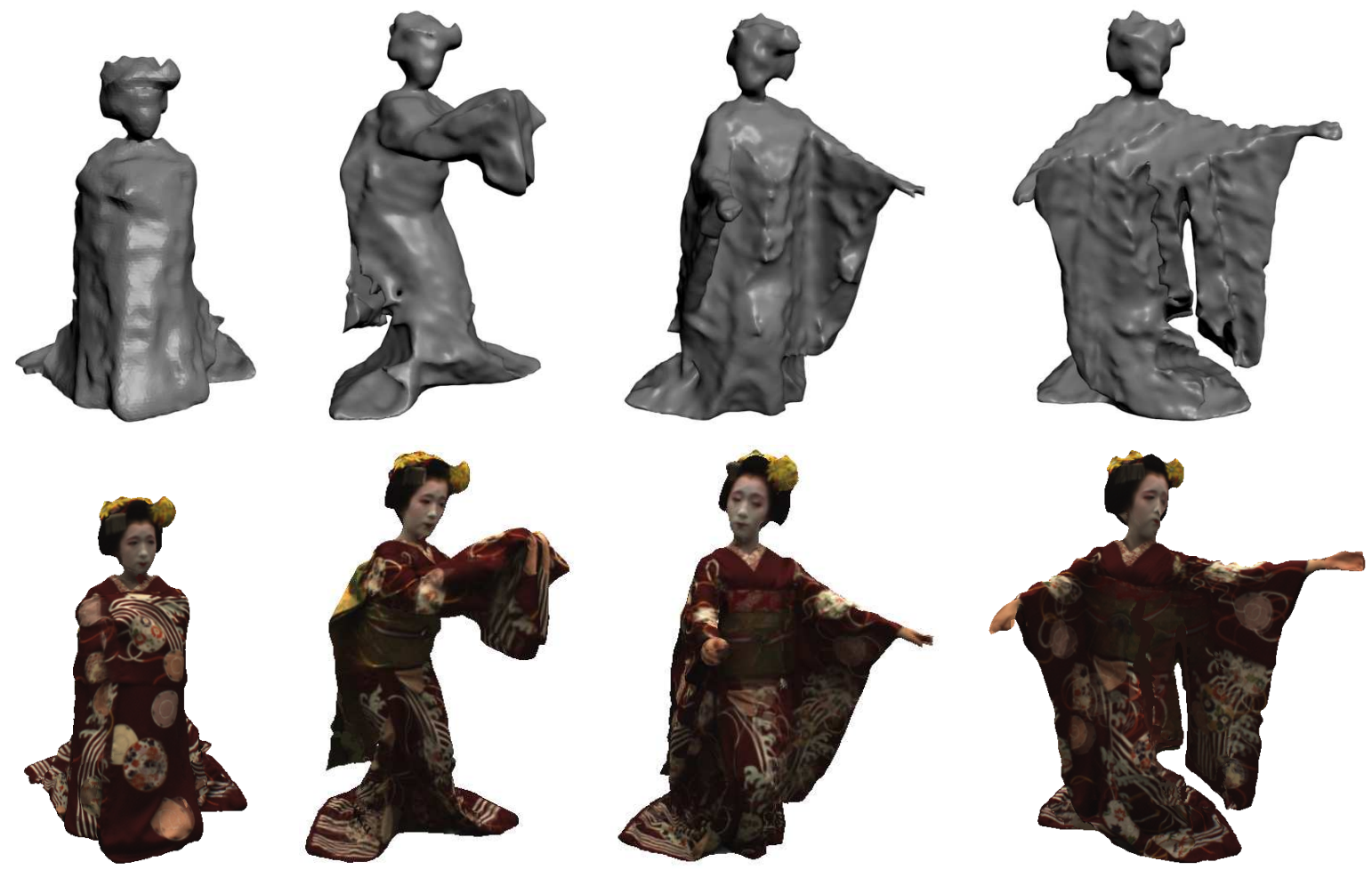

(a) Reconstruction using shape optimisation [3] and view-dependent rendering.
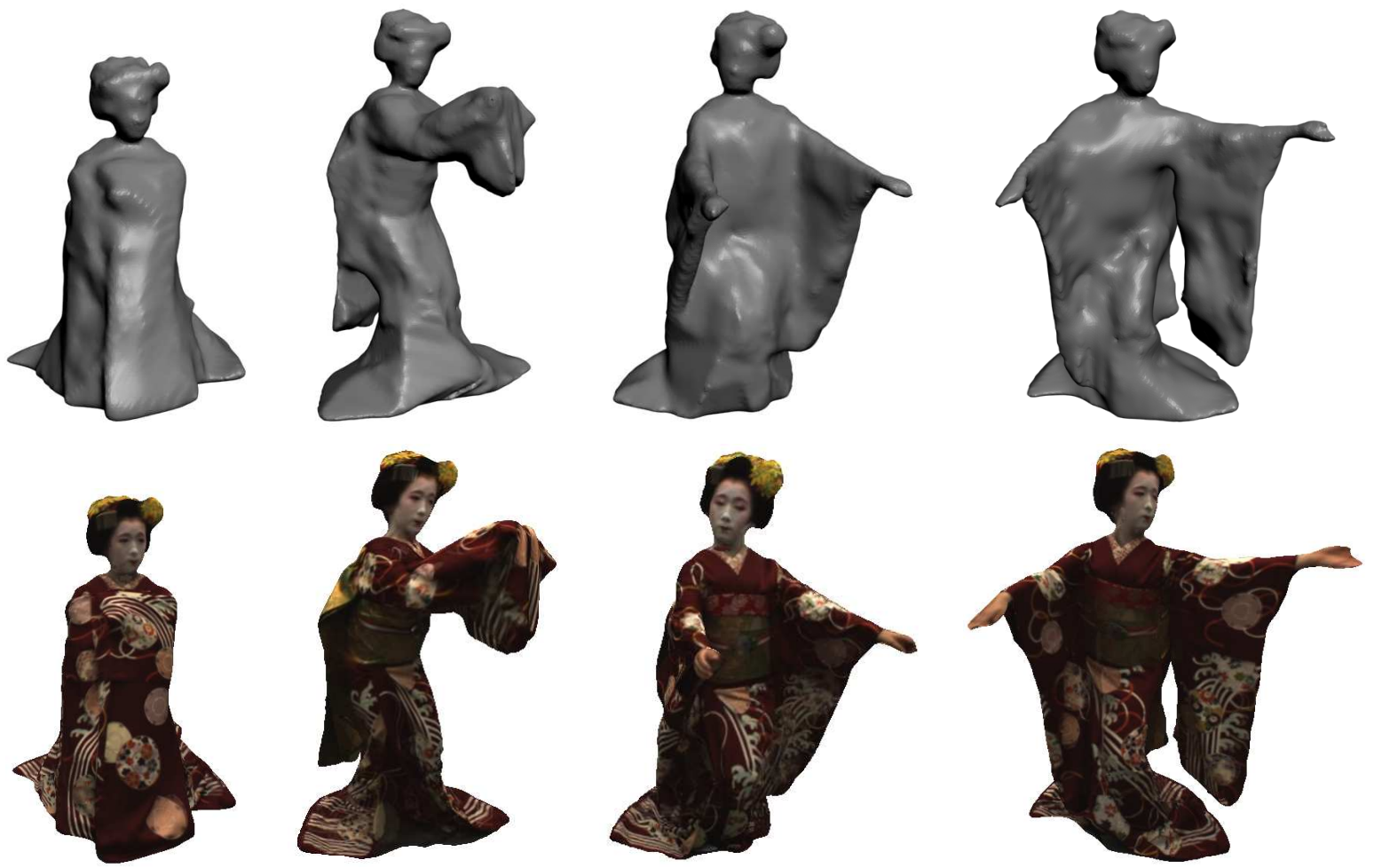

(b) Reconstruction using global optimisation [2] and view-dependent rendering.

Fig. 13. Comparison for the kimono sequence recorded at Kyoto University. 
the multiple camera capture system. The following conclusions are made based on the practical experience in the two studios and the ground truth evaluation.

The studio backdrop should be chosen to give the maximum contrast with the foreground scene for accurate foreground matting with the minimum impact of the content of the scene. The maximum amount of consistent ambient illumination is required in the studio to allow for a fast camera shutter speed to reduce motion blur and a small aperture to increase the depth of field in the scene. Lights should ideally be placed out of the field of view of the cameras to prevent saturation and blooming. Accurate geometric camera calibration is a prerequisite for $3 \mathrm{D}$ shape reconstruction and photometric correction should be performed between cameras to match the sampled appearance between camera viewpoints.

High-quality professional cameras and lenses are required to maximise the sampling resolution to increase geometric accuracy, surface sampling rate and dynamic range for a scene, however this comes at an increase in cost. Low-cost machine vision cameras can be used to provide more cameras for a given budget. With more cameras a restricted field of view can then be used to increase the effective sampling rate for the scene. However, the number of cameras may be significant as the entire scene must be sampled within the depth of field for the cameras.

Cameras can be placed in an overhead position to remove opposing cameras and overhead lighting from the field of view. Reducing the camera height has relatively little effect on visibility and surface appearance can only be completed by including low level cameras with the disadvantage of including the equipment and lighting within the field of view of the camera system. Fixed cameras provide coverage of a predefined limited capture volume. The capture volume can be extended using multiple camera sets or through an active vision system using object tracking.

The ideal baseline between camera views for both stereo reconstruction and shape-from-silhouette was found to be 25 degrees. Non-coplanar cameras improve the reconstruction accuracy for shape-fromsilhouette. Where the entire scene is within the field of view this corresponds to 16 cameras. Widerbaseline configurations up to 45 degrees are however feasible using global optimisation techniques.

Ground-truth experiments demonstrate that the median image matching accuracy using stereo correspondence is in the order of the pixel size for the camera images. This provides a rule-of-thumb formula for reconstruction accuracy. With current generation cameras and whole-body images of people the achievable accuracy is in the order of $5-10 \mathrm{~mm}$. Accuracy will only be improved by increasing the resolution of the camera system or using multiple camera sets with a restricted field-of-view to increase the sampling rate in the scene.

A $5-10 \mathrm{~mm}$ accurate reconstruction is insufficient to recover the detailed geometry at the face, hands 
and fine cloth wrinkles. With limited accuracy the goal of performance capture should be to recover a sufficiently accurate representation to synthesise content without artefacts. With a fixed surface appearance this can require a reduction in the output resolution for the synthesised view. However, view dependent rendering will allow synthesis at the sampling rate of the original camera images but with some reduction in quality due to blurring of the appearance sampled in different camera views.

In summary, where the number of cameras is restricted the ideal configuration would make use of 16 cameras positioned in a non-planar configuration surrounding a capture area. Lighting can be placed overhead to provide an even ambient illumination. Cameras should be positioned at mid-height level subject to excluding the lighting from the field-of-view and handling opposite facing cameras within the field-of-view. Cameras should be selected to maximise the sampling resolution of the scene. The feasible capture volume is constrained by the depth-of-field that can be achieved with the cameras and lighting. Visualisation then requires view dependent rendering where the output display resolution mirrors the camera resolution.

\section{ACKNOWLEDGMENT}

This work was supported by the Daiwa Anglo-Japanese Foundation, EPSRC UK Visual Media Research Platform Grant GR/S46543 and the MEXT Japan Leading Project Grant in Development of High Fidelity Digitization Software for Large-Scale and Intangible Cultural Assets.

\section{REFERENCES}

[1] T. Kanade, P. Rander, and P. Narayanan, "Virtualized reality: Constructing virtual worlds from real scenes," IEEE Multimedia, vol. 4(1), pp. 34-47, 1997.

[2] J. Starck and A. Hilton, "Surface capture for performance based animation," IEEE Computer Graphics and Applications, vol. 27(3), pp. 21-31, 2007.

[3] T. Matsuyama, X. Wu, T. Takai, and S. Nobuhara, "Real-time 3D shape reconstruction, dynamic 3D mesh deformation, and high fidelity visualization for 3D video," Computer Vision and Image Understanding, vol. 96(3), pp. 393-434, 2004.

[4] T. Moeslund, A. Hilton, and V. Kruger, "A survey of advances in video-based human motion capture and analysis," Computer Vision and Image Understanding, vol. 104, pp. 90-126, 2006.

[5] J. Starck and A. Hilton, "Model-based multiple view reconstruction of people," IEEE International Conference on Computer Vision (ICCV), pp. 915-922, 2003.

[6] J. Carranza, C. M. Theobalt, M. Magnor, and H. Seidel, "Free-viewpoint video of human actors," ACM Transactions on Graphics (SIGGRAPH), vol. 22(3), pp. 569-577, 2003.

[7] C. Theobalt, G. Ziegler, M. Magnor, and H.-P. Seidel, "Model-based free-viewpoint video: Acquisition, rendering, and encoding," Picture Coding Symposium (PCS), vol. 5, pp. 1-6, 2004. 
[8] P. Sand, L. McMillan, and J. Popovic, "Continuous capture of skin deformation," ACM Transactions on Graphics (SIGGRAPH), pp. 578-586, 2003.

[9] A. Balan, L. Sigal, M. Black, J. Davis, and H. Haussecker, "Detailed human shape and pose from images," to appear Computer Vision and Pattern Recognition (CVPR), 2007.

[10] S. Seitz, B. Curless, J. Diebel, D. Scharstein, and R. Szeliski, "A comparison and evaluation of multi-view stereo reconstruction algorithms," IEEE International Conference on Computer Vision and Pattern Recognition (CVPR), vol. 1, pp. 519-526, 2006.

[11] A. Laurentini, "The visual hull concept for silhouette based image understanding," IEEE Transactions on Pattern Analysis and Machine Intelligence, vol. 16(2), pp. 150-162, 1994.

[12] K. Kutulakos and S. Seitz, "A theory of shape by space carving," International Journal of Computer Vision, vol. 38(3), pp. 199-218, 2000.

[13] O. Faugeras and R. Keriven, "Variational principles, surface evolution, pde's, level set methods and the stereo problem," IEEE Transactions on Image Processing, vol. 7(3), pp. 336-344, 1998.

[14] B. Goldluecke and M. Magnor, "Space-time isosurface evolution for temporally coherent 3D reconstruction," IEEE International Conference on Computer Vision and Pattern Recognition (CVPR), vol. I, pp. 350-355, 2004.

[15] C. Esteban and F. Schmitt, "Silhouette and stereo fusion for 3D object modeling," Computer Vision and Image Understanding, vol. 96(3), pp. 367-392, 2004.

[16] J. Starck and A. Hilton, "Virtual view synthesis of people from multiple view video sequences," Graphical Models, vol. 67(6), pp. 600-620, 2005.

[17] Y. Furukawa and J. Ponce, "Carved visual hulls for image-based modeling," European Conference on Computer Vision (ECCV), pp. I: 564-577, 2006.

[18] M. Levoy and P. Hanrahan, "Light field rendering," ACM Transactions on Graphics (SIGGRAPH), vol. 30, pp. 31-42, 1996.

[19] P. Debevec, Y. Yu, and G. Borshukov, "Efficient view-dependent image-based rendering with projective texture-mapping," Proceedings of Eurographics Workshop on Rendering,, pp. 105-116, 1998.

[20] C. Buehler, M. Bosse, L. McMillan, S. Gortler, and M. Cohen, "Unstructured lumigraph rendering," ACM Transactions on Graphics (SIGGRAPH), pp. 425-432, 2001.

[21] C. Zitnick, S. B. Kang, M. Uyttendaele, S. A. J. Winder, and R. Szeliski, "High-quality video view interpolation using a layered representation," ACM Transactions on Graphics (SIGGRAPH), vol. 23(3), pp. 600-608, 2004.

[22] G. Cheung, T. Kanade, J. Bouguet, and M. Holler, "A real time system for robust 3D voxel reconstruction of human motions," IEEE International Conference on Computer Vision and Pattern Recognition (CVPR), pp. 714-720, 2000.

[23] W. Matusik, C. Buehler, and L. Mcmillan, "Polyhedral visual hulls for real-time rendering," Proceedings of Eurographics Workshop on Rendering, pp. 115-126, 2001.

[24] O. Grau, T. Pullen, and G. Thomas, "A combined studio production system for 3D capturing of live action and immersive actor feedback," IEEE Transactions on Circuits and Systems for Video Technology, vol. 14(3), pp. 370-380, 2003.

[25] J.-M. Hasenfratz, M. Lapierre, and F. Sillion, "A real-time system for full body interaction with virtual worlds," Eurographics Symposium on Virtual Environments, pp. 147-156, 2004.

[26] J. Allard, J.-S. Franco, C. Menier, E. Boyer, and B. Raffin, "The grimage platform: A mixed reality environment for interactions," IEEE International Conference on Computer Vision Systems (ICVS), p. 46, 2006. 
[27] C. Theobalt, M. Li, M. Magnor, and H.-P. Seidel, “A flexible and versatile studio for synchronized multi-view video recording," Vision, Video, and Graphics, pp. 9-16, 2003.

[28] T. Kanade, H. Saito, and S. Vedula, "Digitizing time-varying 3D events by synchronized multiple video streams," Technical Report CMU-RI-TR-98-34, Robotics Institute, Carnegie Mellon University, 1998.

[29] K. Cheung, S. Baker, and T. Kanade, "Silhouette across time: Part ii: Applications to human modeling and markerless motion tracking," International Journal of Computer Vision, vol. 63(3), pp. 225-245, 2005.

[30] C. Zhang and T. Chen, "A self-reconfigurable camera array," Eurographics Symposium on Rendering, pp. 243-254, 2004.

[31] W. Matusik and H. Pfister, "3D TV: a scalable system for real-time acquisition, transmission, and autostereoscopic display of dynamic scenes," ACM Transactions on Graphics (SIGGRAPH), pp. 814-824, 2004.

[32] M. Tanimoto, "FTV (free viewpoint television) for 3D scene reproduction and creation," IEEE Conference on Computer Vision and Pattern Recognition Workshop (CVPRW), p. 172, 2006.

[33] M. Waschbusch, S. Wurmlin, D. Cotting, F. Sadlo, and M. Gross, "Scalable 3D video of dynamic scenes," The Visual Computer, vol. 21(8-10), pp. 629-638, 2005.

[34] J.-S. Franco, M. Lapierre, and E. Boyer, "Visual shapes of silhouette sets," International Symposium on 3D Data Processing, Visualization and Transmission (3DPVT), pp. 397-404, 2006.

[35] K. Tomiyama, Y. Orihara, M. Katayama, and Y. Iwadate, "Algorithm for dynamic 3D object generation from multi-viewpoint images," Proceedings of the SPIE, vol. 5599(19), pp. 153-161, 2004.

[36] Y. Boykov and V. Kolmogorov, "Computing geodesics and minimal surfaces via graph cuts," IEEE International Conference on Computer Vision (ICCV), pp. 26-33, 2003.

[37] S. Vedula, S. Baker, and T. Kanade, "Image-based spatio-temporal modeling and view interpolation of dynamic events," ACM Transactions on Graphics, vol. 24(2), pp. 240-261, 2005.

[38] Y.-Y. Chuang, B. Curless, D. Salesin, and R. Szeliski, "A Bayesian approach to digital matting," IEEE Conference on Computer Vision and Pattern Recognition (CVPR), pp. 264-271, 2001.

[39] N. Ukita and T. Matsuyama, "Real-time cooperative multi-target tracking by communicating active vision agents," Computer Vision and Image Understanding, vol. 97(2), pp. 137-179, 2005.

[40] R. Tsai, "An efficient and accurate camera calibration technique for 3D machine vision," IEEE Conference on Computer Vision and Pattern Recognition (CVPR), pp. 364-374, 1986.

[41] Z. Zhang, "A flexible new technique for camera calibration," IEEE Transactions on Pattern Analysis and Machine Intelligence, vol. 22(11), pp. 1330-1334, 2000.

[42] R. I. Hartley and A. Zisserman, Multiple View Geometry in Computer Vision, 2nd ed. Cambridge University Press, 2004.

[43] T. Svoboda, D. Martinec, and T. Pajdla, "A convenient multicamera self-calibration for virtual environments," Presence: Teleoper. Virtual Envrion, vol. 14(4), pp. 407-422, 2005.

[44] J. Mitchelson and A. Hilton, "Wand-based multiple camera studio calibration," CVSSP Technical Report, vol. VSSP-TR2/2003, 2003.

[45] A. Wenger, T. Hawkins, and P. Debevec, "Optimizing color matching in a lighting reproduction system for complex subject and illuminant spectra," Eurographics workshop on Rendering, pp. 249-259, 2003.

[46] P. Cignoni, C. Rocchini, and R. Scopigno, "Metro: measuring error on simplified surfaces," Computer Graphics Forum, vol. 17(2), pp. 167-174, 1998.

[47] O. Faugeras, B. Hotz, H. Mathieu, T. Vieville, Z. Zhang, P. Fua, E. Theron, L. Moll, G. Berry, J. Vuillemin, P. Bertin, and C. Proy, "Real time correlation based stereo: algorithm implementations and applications," INRIA Report $2013,1993$. 
[48] A. Fusiello, E. Trucco, and A. Verri, "Rectification with unconstrained stereo geometry," British Machine Vision Conference (BMVC), pp. 400-409, 1997.

[49] A. Fusiello, V. Roberto, and E. Trucco, "Symmetric stereo with multiple windowing," International Journal of Pattern Recognition and Artificial Intelligence, vol. 14(8), pp. 1053-1066, 2000.

[50] A. R. Greenleaf, "Photographic optics," The MacMillan Company, New York, pp. 25-27, 1950. 